\title{
Review of diffraction at HERA and Tevatron
}

\author{
Laurent Schoeffel \\ CEA Saclay, DAPNIA-SPP, 91191 Gif-sur-Yvette Cedex, France
}

We present and discuss the recent results on diffraction from the HERA and Tevatron experiments.

\section{DIFFRACTION AT HERA}

At low $x$ in deep inelastic scattering (DIS) at HERA, approximately $10 \%$ of the events are of the type $e p \rightarrow e X p$, where the final state proton carries more than $95 \%$ of the proton beam energy [1]. For these processes, a photon of virtuality $Q^{2}$, coupled to the electron (or positron), undergoes a strong interaction with the proton (or one of its low-mass excited states $Y$ ) to form a hadronic final state system $X$ of mass $M_{X}$ separated by a large rapidity gap (LRG) from the leading proton (see Fig. 1). These events are called diffractive. In such a reaction, $e p \rightarrow e X p$, no net quantum number is exchanged and the longitudinal momentum fraction $1-x_{\mathbb{P}}$ is lost by the proton. Thus, the mongitudinal momentum $x_{\mathbb{P}} P$ is transfered to the system $X$. In addition, the virtual photon couples to a quark carrying a fraction $\beta=x_{B j} / x_{\mathbb{P}}$ of the exchanged momentum, where $x_{B j}$ is the Bjorken variable. Extensive measurements of diffractive DIS cross sections have been made by both the ZEUS and H1 collaborations at HERA, using different experimental techniques 2]. The results are presented in Fig. 2.

Events with the diffractive topology can be analysed in Regge models in terms of Pomeron trajectory exchanged between the proton and the virtual photon. Then, these events result from a colour-singlet exchange between the diffractively dissociated virtual photon and the proton. Since this phenomenon is present even for high virtuality of the photon, it is called hard diffraction, at variance with soft diffractive phenomena. Several theoretical formulations have been proposed.

Among the most popular models, the one based on a pointlike structure of the Pomeron has been studied quantitatively using a non-perturbative input supplemented by a perturbative QCD evolution equations [3]. In this formulation, it is assumed that the exchanged object, the Pomeron, is a colour-singlet quasiparticle whose structure is probed in DIS process. As for standard DIS, diffractive parton distributions (PDFs) related to the Pomeron can be derived from QCD fits to diffractive cross sections [3]. Diffractive PDFs extracted from $\mathrm{H} 1$ and ZEUS data are shown in Fig. 3. We notice the large uncertainty (of about $25 \%$ ) at large values of $\beta$ using different data sets [3].

There exists a different approach in which the cross sections are determined by the interaction between colour dipole states describing the photon and the proton [4]. Indeed, it is well-known that the pho-

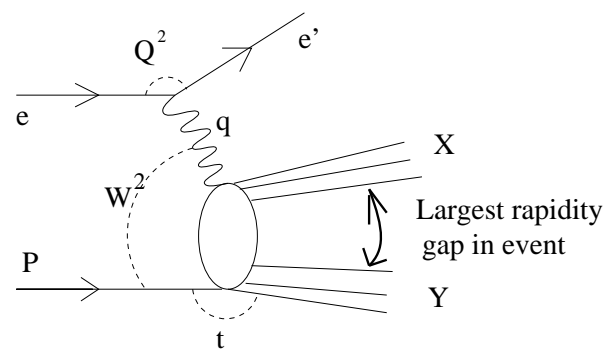

FIG. 1: Illustration of the process $e p \rightarrow e X Y$. The hadronic final state is composed of two distinct systems $X$ and $Y$, which are separated by the largest interval in rapidity between final state hadrons.

ton can be analyzed in terms of $q \bar{q}$ configurations while it has been shown that the small- $x_{B j}$ structure functions of the proton can be described by a collection of primordial dipoles with subsequent perturbative QCD evolution. This approach leads to a unified description of the proton total and diffractive structure functions and provides a natural explanation for the geometric scaling property observed on the data [5, 6]. In Fig. 4, diffractive cross sections, $\beta \frac{d \sigma_{d i f f}^{\gamma^{*} p \rightarrow X p}}{d \beta}=\frac{4 \pi^{2} \alpha_{e m}}{Q^{2}} x_{\mathbb{P}} F_{2}^{D(3)}$, are presented as a fonction of the scaling variable $\tau_{d}=Q^{2} / Q_{s}^{2}$, where $Q_{s}^{2}$ is the saturation scale combining variables $Q^{2}, x$ and $x_{\mathbb{P}}$ as defined in Ref. [6]. For each values of $\beta$, the function $\beta \frac{d \sigma_{d i f f}^{\gamma^{*} p \rightarrow X}}{d \beta}\left(\tau_{d}\right)$ is then observed to be continuous : it defines what is called the geometrical scaling behaviour in this context [6].

\section{DIFFRACTION AT TEVATRON AND LHC}

The difference between diffraction at HERA and at the Tevatron is that diffraction at the Tevatron can occur not only on either $p$ or $\bar{p}$ side as at HERA, but also on both sides. The former case is called single diffraction and the other one, double Pomeron exchange. In the same way as we have defined the kinematical variables $x_{\mathbb{P}}$ and $\beta$ at HERA, we define $\xi_{1,2}$ as the protons fractional momenta loses and $\beta_{1,2}$, the fractions of the Pomeron momentum carried by the interacting partons. The produced diffractive mass is equal to $M^{2}=s \xi_{1}$ for single diffractive events and to $M^{2}=s \xi_{1} \xi_{2}$ for double Pomeron exchange, where $\sqrt{s}$ is the energy of the reaction in the center of mass frame. The size of the rapidity gap is then of the order 


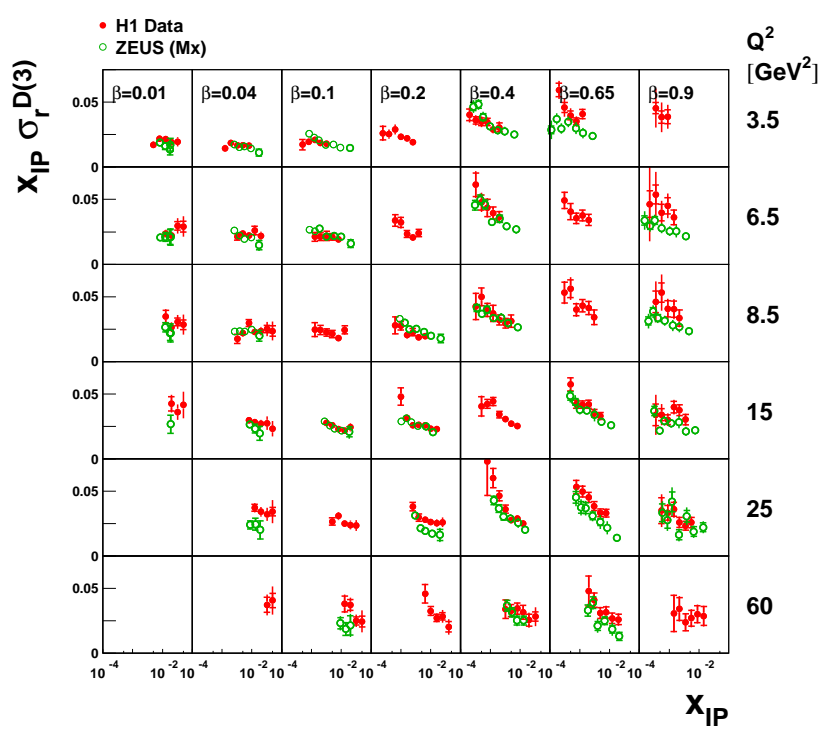

FIG. 2: Diffractive structure function measurements of the $\mathrm{H} 1$ and ZEUS collaborations at HERA. The observable $\sigma_{r}^{D(3)} \simeq F_{2}^{D(3)}$ is determined from the measured cross section using the relation : $\frac{d^{3} \sigma^{e p \rightarrow e X p}}{d x_{\mathbb{P}} d x d Q^{2}}=$ $\frac{4 \pi \alpha_{e m}^{2}}{x Q^{4}}\left(1-y+\frac{y^{2}}{2}\right) \sigma_{r}^{D(3)}\left(x_{\mathbb{P}}, x, Q^{2}\right)$.
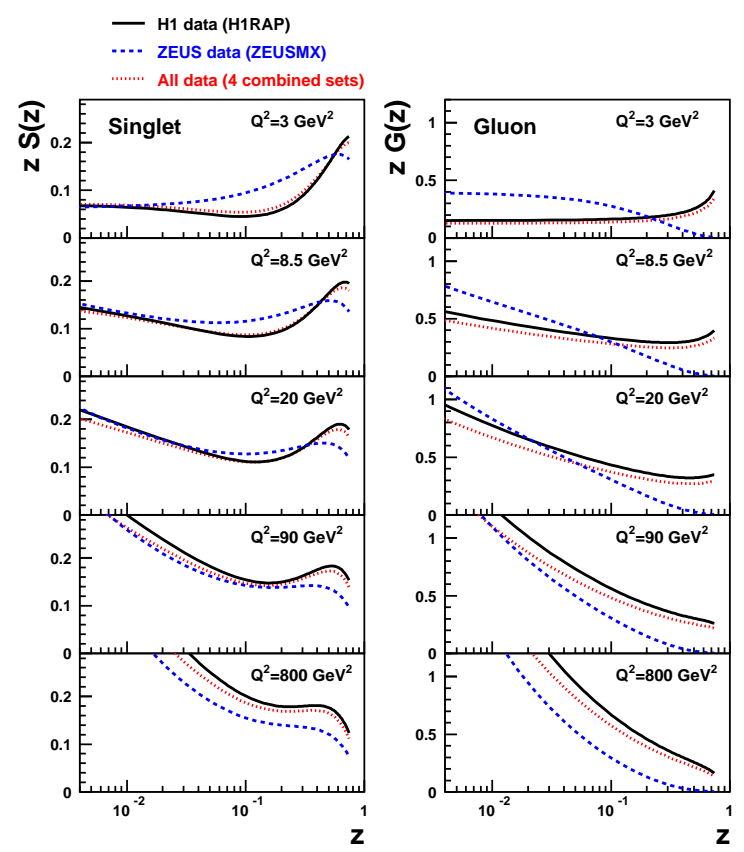

FIG. 3: Singlet and gluon distributions of the Pomeron as a function of $z \equiv \beta$, the fractional momentum of the Pomeron carried by the struck parton (see text) [3] .

of $\Delta \eta \sim \log 1 / \xi_{1,2},[1,7]$.

It has been shown that the diffractive PDFs of HERA can not be used directly to make predictions at the Tevatron. Indeed, factorisation does not hold and a gap survival probability of a few $\%$ has to be

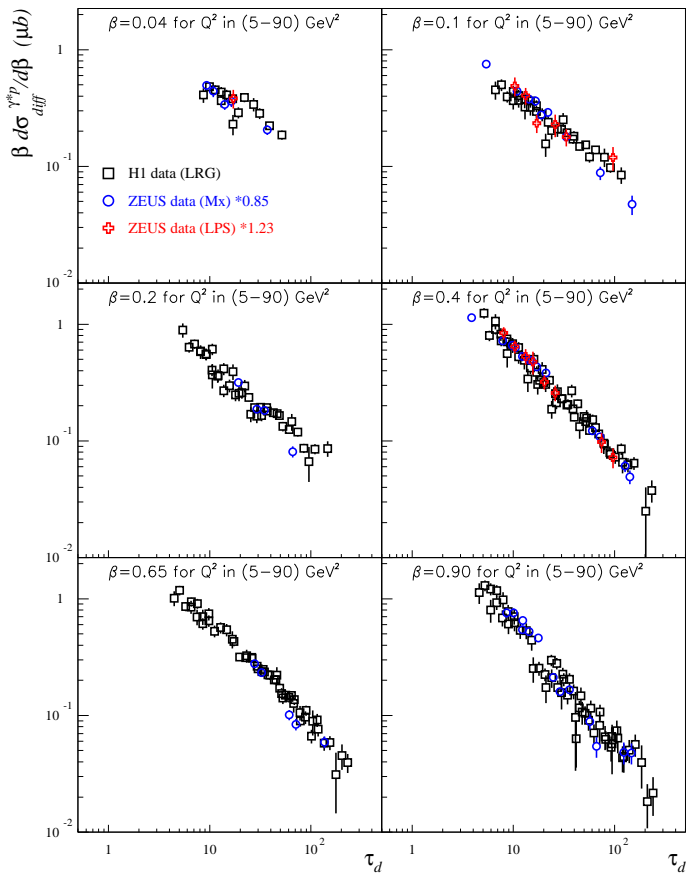

FIG. 4: The diffractive cross section $\beta d \sigma_{d i f f}^{\gamma^{*} p \rightarrow X p} / d \beta$ from $\mathrm{H} 1$ and ZEUS measurements, as a function of $\tau_{d}$ in bins of $\beta$ for $Q^{2}$ values in the range $[5 ; 90] \mathrm{GeV}^{2}$ and for $x_{\mathbb{P}}<0.01$ (see text) [6].

considered (see Fig. 5). It corresponds to the probability that there is no soft additional interaction or in other words that the event remains diffractive [1, 7].

The CDF collaboration has measured the so-called dijet mass fraction (DMF) in dijet events when the antiproton is tagged in the roman pot detectors and when there is a rapidity gap on the proton side to ensure that the event corresponds to a double Pomeron exchange. The measured observable $R_{j j}$ is defined as the ratio of the mass carried by the two jets divided by the total diffractive mass [1, 7, 8]. The DMF turns out to be a very appropriate observable for identifying the exclusive production, which would manifest itself as an excess of the events towards $R_{j j} \sim 1[1,7,8]$. Indeed, for exclusive events, the dijet mass is essentially equal to the mass of the central system because no Pomeron remnant is present. Then, for exclusive events, the DMF is 1 at generator level and can be smeared out towards lower values taking into account the detector resolutions. The advantage of the DMF is that one can focus on the shape of the distribution and let the absolute normalisation of the predictions as free parameters (in a first step). Indeed, the observation of exclusive events does not rely on the overall normalization which might be strongly dependent on the detector simulation and acceptance of the roman pot detector. Results are shown in Fig 6 with Monte-Carlo (MC) expectations. These MC predic- 


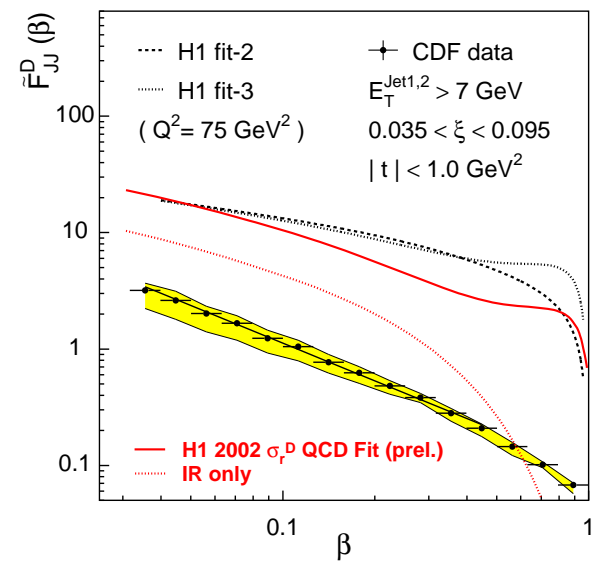

FIG. 5: Comparison between the CDF measurement of diffractive structure function (black points) with the expectation of the HERA diffractive PDFs.

tions are displayed for inclusive double Pomeron exchange events, where remnants are still present in the reaction and then $R_{j j}$ is lower that unity, as well as for exclusive events in a specific model. More details are given on the models entering into these simulations in Ref. [1, 8]. We observe a good agreement when both contributions, inclusive and exclusive, are taken into account. A MC with inclusive simulation only is not sufficient to describe the present data, and shows a clear deficit of events towards high values of the DMF, where exclusive events are supposed to occur. This part of the DMF is properly described by the exclusive contribution, once normalised to fit the histogram. It is a first evidence that exclusive events could contribute at the Tevatron [1, 8]].

The great interest of studying such exclusive dijet events is that it opens the possibility to analyse the production of heavy objects in double Pomeron exchange at the LHC [1, 7, 8]. In particular, the production of a Higgs boson in such a topology could be quite interesting as the event would be very clean : both protons escape and are detected in Roman pots, two large rapidity gaps on both sides and the central production of the Higgs boson, leading to some decay products well isolated in the detector $[1,6$, 8 ]. The major advantage of such events is that the resolution on the mass of the produced object can be determined with a high resolution from the measurement of the proton momentum loses, using the relation $M^{2}=s \xi_{1} \xi_{2}$. A potential signal, accessible in a mass distribution, is then not washed out by the lower resolution when using central detectors, rather than forward Roman pots to measure $\xi_{1}$ and $\xi_{2}$.

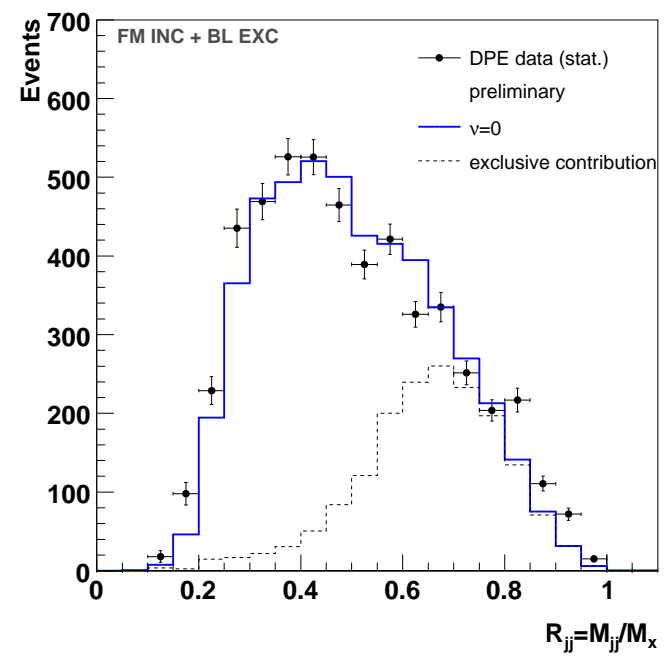

FIG. 6: Dijet mass fraction for jets $p_{T}>10 \mathrm{GeV}$. The data are compared to the sum of inclusive and exclusive predictions. The dPDFs derived from $\mathrm{H} 1$ data have been used together with the survival gap probability measured with single diffractive events at Tevatron.

\section{CONCLUSIONS}

We have presented and discussed the most recent results on inclusive diffraction from the HERA and Tevatron experiments. Of special interest for future prospects is the exclusive production heavy objects (including Higgs boson) which requires a good understanding of diffractive processes and the link between electron-proton and hadronic colliders.
[1] C. Royon, Acta Phys. Polon. B 37 (2006) 3571.

[2] A. Aktas et al. [H1 Collaboration], Eur. Phys. J. C 48 (2006) 715; S. Chekanov et al. [ZEUS Collaboration], Nucl. Phys. B713 (2005) 3.

[3] C. Royon, L. Schoeffel, S. Sapeta, R. Peschanski and E. Sauvan, arXiv:hep-ph/0609291; Nucl. Phys. B 746 (2006) 15.

[4] A. Bialas and R. Peschanski, Phys. Lett. B378 (1996) 302; S. Munier, R. Peschanski and C. Royon, Nucl. Phys. B534 (1998) 297.
[5] K. Golec-Biernat and M. Wüsthoff, Phys. Rev. D59 (1999) 014017; Phys. Rev. D60 (1999) 114023.

[6] C. Marquet and L. Schoeffel, Phys. Lett. B 639 (2006) 471.

[7] L. Schoeffel, proceedings Blois 2007, arXiv:0706.3484 [hep-ph]; proceedings Moriond QCD 2007, arXiv:0705.1413 [hep-ph].

[8] O. Kepka and C. Royon, arXiv:0704.1956 [hep-ph]. 